\title{
Research Article \\ Balance Performance Is Task Specific in Older Adults
}

\author{
Ayelet Dunsky, Aviva Zeev, and Yael Netz \\ The Academic College at Wingate, Wingate Institute, Netanya, Israel \\ Correspondence should be addressed to Yael Netz; neyael@wincol.ac.il \\ Received 7 May 2017; Accepted 3 August 2017; Published 5 September 2017 \\ Academic Editor: Javier Olazarán
}

Copyright (C) 2017 Ayelet Dunsky et al. This is an open access article distributed under the Creative Commons Attribution License, which permits unrestricted use, distribution, and reproduction in any medium, provided the original work is properly cited.

\begin{abstract}
Balance ability among the elderly is a key component in the activities of daily living and is divided into two types: static and dynamic. For clinicians who wish to assess the risk of falling among their elderly patients, it is unclear if more than one type of balance test can be used to measure their balance impairment. In this study, we examined the association between static balance measures and two dynamic balance field tests. One hundred and twelve community-dwelling older adults (mean age 74.6) participated in the study. They underwent the Tetrax static postural assessment and then performed the Timed Up and Go (TUG) and the Functional Reach (FR) Test as dynamic balance tests. In general, low-moderate correlations were found between the two types of balance tests. For women, age and static balance parameters explained $28.1-40.4 \%$ of the variance of TUG scores and $14.6-24 \%$ of the variance of FR scores. For men, age and static balance parameters explained $9.5-31.2 \%$ of the variance of TUG scores and $23.9-41.7 \%$ of the variance of FR scores. Based on our findings, it is suggested that a combination of both static and dynamic tests be used for assessing postural balance ability.
\end{abstract}

\section{Introduction}

The ability to control balance is based on the integration of sensory information from the somatosensory, vestibular, and visual systems, which work together with the nervousmuscular system to control body alignment with respect to the environment and to stabilize the body's center of mass during perturbations, with subsequent motor output.

Balance is considered a key component in many activities of daily living, from simple activities such as quiet standing, to more complex activities such as walking while talking or while changing directions [1]. These different tasks require different components of balance ability, which are usually divided into two types of balance: static and dynamic. Static balance is defined as the ability to maintain an upright posture and to keep the line of gravity within the limits of the base of support (i.e., quiet standing) [2]. Dynamic balance is defined as the ability to maintain stability during weight shifting, often while changing the base of support [1].

The ability to control balance deteriorates with age as a result of alternations in the vestibular, visual, somatosensory, musculoskeletal, and central nervous systems [3]. In addition, many older people with balance disorders suffer from orthopedic constraints and cognitive impairments [4]. As a consequence, many people in the elderly population show impairments in stability and balance and thus are subjected to a risk of falling in both static and dynamic situations [4-6].

Detection of the level of impaired postural control is important for the assessment of risk of falling, as well as for the evaluation of proper treatment [7]. Therefore, researchers that study the association between balance and falling, or those who examine the effects of an exercise program on balance, use different balance tests that include static or dynamic aspects [8]. As static and dynamic balance controls are based on the integration and coordination of the same systems, some researchers assess only one type of balance control and consider this assessment is the sole measure of balance ability $[5,9]$. However, other researchers assess both types of balance and find different levels of performance between them $[7,10]$. This discrepancy led researchers to study the relationship between static and dynamic balance ability. For example, Carter et al. [11] showed moderate correlation $(r=0.59)$ between the two types of balance abilities, Mayson et al. [12] reported a range of correlation values between low to moderate $(r=0.19-0.60)$ for different static and dynamic balance tasks, Shimada et al. [13] found almost 
no relationships between standing balance tests and dynamic balance control during perturbed walking, and Muehlbauer et al. [14] found no significant correlations between the two types of balance.

Consequently, it is currently unclear to what extent static balance ability and dynamic balance ability among adults are correlated. Thus, for clinicians who wish to assess the risk of falling among their elderly patients, it is unclear if using only one type of balance test is adequate as a measure of their balance impairment. Furthermore, if balance is comprised of various unrelated skills, clinicians should develop training programs that will stimulate all of these skills. In this study, we examine the association between a number of static balance measures and two prevalent dynamic balance field tests.

\section{Materials and Methods}

2.1. Participants. One hundred and twenty-three communitydwelling older adults, age 65 and over, volunteered to participate in the study. They were recruited from the general community by ads that appeared on Facebook or were distributed in several sports clubs and in local councils located around the Wingate Institute (where the study was conducted). Inclusion criteria were as follows: being able to perform and complete a maximal exercise test and being involved in habitual physical activity at least once a week for at least three months prior to the commencement of the study. Exclusion criteria included a score of $<24$ on the Mini Mental State Examination (MMSE) [15] and risk factors of health problems raised by the physician. Based on the decision of the physician, eleven volunteers were excluded (mainly due to cardiovascular risk factors); thus the results reported here represent data from 112 participants. Informed consent was obtained from all participants on a consent form approved by the Institutional Review Board of the Hillel Yaffe Medical Center (Hadera, Israel).

2.2. Procedure. Participants visited the laboratory twice in one week. At their first assessment session, participants completed a demographic questionnaire, the Instrumental Activities Daily Living (IADL) questionnaire [16], the short version of the Geriatric Depression Scale (GDS) [17], and the MMSE [15]. The demographic details and mean scores of the clinical assessments are presented in Table 1. Then, two dynamic balance assessments were performed. In the second visit the static balance assessments were performed.

2.2.1. Dynamic Balance Assessments. The Timed Up and Go (TUG) [18], the length of time it takes to stand up from a seated position in a chair, walk three meters, walk around a plastic cone, and return to a seated position, measured in seconds. Lower values indicate better balance ability. The TUG is considered to be a measure of dynamic balance as well as a measure of general mobility, since it represents a sum of transitions of the body, including changes of direction and different heights of the center of mass. The test was performed twice. The better score (lower value) was used for data analysis.
TABle 1: Demographic and clinical description of the participants (mean \pm standard deviations).

\begin{tabular}{lcc}
\hline & Women & Men \\
\hline$N$ & 86 & 36 \\
Age (years) & $74(6)$ & $77(5)$ \\
Weight $(\mathrm{kg})$ & $68.43(10.74)$ & $77.89(9.74)$ \\
Height $(\mathrm{m})$ & $1.58(0.06)$ & $1.72(0.06)$ \\
BMI $\left(\mathrm{kg} / \mathrm{m}^{2}\right)$ & $27.43(3.98)$ & $26.36(3.50)$ \\
MMSE & $29.15(1.30)$ & $28.69(1.83)$ \\
GDS & $2.27(2.47)$ & $1.67(2.04)$ \\
Education (years) & $12.85(3.31)$ & $14.03(3.17)$ \\
Lawton & $1.12(0.20)$ & $1.08(0.22)$ \\
Physical activity (total & $429.76(218.63)$ & $577.94(256.78)$ \\
min/week) & &
\end{tabular}

The Functional Reach Test (FR) [19], the distance an individual can reach forward beyond arm's length while maintaining a standing fixed base of support, measured in centimeters. Higher values indicate a longer maximal safe standing forward reach. The test was performed twice. The higher score was used for data analysis.

2.2.2. Static Balance Assessment. The assessment of postural control was carried out by the Tetrax ${ }^{\circledR}$ posturography device (BeamMed Ltd. [Sunlight], Petah Tikva, Israel). The Tetrax consists of two pairs of single piezoelectric sensor platforms that record posturographic right and left heel and toe forces applied to the ground. The analysis is based on the vertical pressure applied via the heels and the toes while standing in an upright position on four plates. Data collection was documented in eight different conditions, each of which reflects the influence of different body systems that affect postural control: (a) normal open position (NO): standing straight, with eyes open; (b) normal closed position (NC): standing straight, with eyes closed; (c) pillows open (PO): standing on pillows, with eyes open; (d) pillows closed (PC): standing on pillows, with eyes closed; (e) head right (HR): standing with the head turned right, with eyes closed; (f) head left (HL): standing with the head turned left, with eyes closed; (g) head back (HB): standing with head tilted backward at a 30-degree angle, with eyes closed; and (h) head forward (HF): standing with head tilted forward about 30 degrees, with eyes closed. For each condition, the participant was instructed to maintain an upright position without moving for 30 seconds [20].

For each condition the Tetrax software computed the following parameters: (1) stability index (ST), the total amount of sway from the four footplates (right and left heels, toes of right and left foot), which was totaled and then divided by the subject's weight. The higher the score, the greater the posture instability. This value correlates strongly with the values "area of sway," "length of sway," and "amount of sway" of other investigational systems; (2) the pattern of sway intensities at different frequency ranges, as shown by Fourier spectral analysis of the postural sway waves. Eight frequency band ranges $(F 1-F 8$, in $\mathrm{Hz})$ were computed for 
TABLE 2: Regression of static balance parameters on TUG, by gender.

\begin{tabular}{|c|c|c|c|c|}
\hline \multirow{2}{*}{ Static balance condition } & \multicolumn{2}{|c|}{ Women } & \multicolumn{2}{|c|}{ Men } \\
\hline & $R^{2}$ total & Variable included & $R^{2}$ total & Variable included \\
\hline $\mathrm{NO}$ & 31.7 & $\operatorname{Age}^{* *}, F 3^{*}$ & 9.5 & \\
\hline $\mathrm{NC}$ & 32.5 & $\operatorname{Age}^{* *}, F 4^{*}$ & 29.6 & \\
\hline $\mathrm{PO}$ & 33.2 & Age $^{* *}$ & 14.5 & \\
\hline $\mathrm{PC}$ & 31.1 & $\operatorname{Age}^{* *}, F 8^{*}$ & 31.2 & $F 3^{*}$ \\
\hline $\mathrm{HR}$ & 40.4 & $\mathrm{Age}^{* *}, F 2^{* *}, F 4^{*}$ & 29.5 & $F 7^{*}$ \\
\hline HL & 28.1 & $\operatorname{Age}^{* *}$ & 20.4 & \\
\hline $\mathrm{HB}$ & 34.2 & $\operatorname{Age}^{* *}, F 2^{*}$ & 16.3 & \\
\hline $\mathrm{HF}$ & 30.2 & $\operatorname{Age}^{* *}, F 8^{*}$ & 26.5 & \\
\hline
\end{tabular}

${ }^{*} p<0.05 ;{ }^{* *} p<0.01$. NO: normal open position: standing straight, with eyes open. NC: normal closed position: standing straight, with eyes closed. PO: pillows open: standing on pillows, with eyes open. PC: pillows closed: standing on pillows, with eyes closed. HR: head right: standing with the head turned right, with eyes closed. HL: head left: standing with the head turned left, with eyes closed. HB: head back: standing with head tilted backward at a 30-degree angle, with eyes closed. HF: head forward: standing with head tilted forward about 30 degrees, with eyes closed. F1-F8 represent the pattern of sway intensities at different frequency ranges, as shown by Fourier spectral analysis of the postural sway waves. $F 1=$ low, $F 2-F 4=$ low-medium, $F 5$ - $F 6=$ medium-high, and $F 7-F 8=$ high frequencies of the Fourier band.

evaluation of the power-frequency pattern, as follows: $F 1=$ low; F2-F4 = low-medium; F5-F6 = medium-high; and F7F8 = high frequencies of the Fourier band. Abnormally high values on $F 1$ may be related to visual dysfunction. Abnormally high values on $F 2-F 4$ may be related to peripheral vestibular dysfunction. Abnormally high values on F5-F6 may be related to somatosensory dysfunction, and abnormally high values on F7-F8 may be related to central dysfunction [20]. A previous study demonstrated reliability of the Tetrax device $\left(\mathrm{ICC}_{2,1}\right)$ with the stability index reported to be 0.850 [21].

2.3. Data Analysis. The analysis was performed in two steps. In the first step, associations between static and dynamic balance were calculated using Pearson's correlation coefficient $(r)$ (see Tables 4 and 5). In the second step, a regression model was used to assess the effect of static balance, in each one of the eight conditions, on dynamic balance. Two indicators of dynamic balance were utilized as dependent variables: the TUG scores and the FR scores. The explanatory variables were the parameters calculated by the Tetrax software: ST and Fourier spectral analysis for each of the static balance conditions that had a significant correlations with the dynamic variable. As age is considered a significant explanatory variable for physical ability, it was also entered to the analysis.

\section{Results}

The mean age for all participants was 74.6 years, and as can be seen in Table 1 they were predominantly women (77\%). The average BMI represents overweighed values for both genders [22]. The IADL questionnaire showed that all participants were independent, and they showed normal values of cognitive functions based on their MMSE results. Most of them had a high school education. Based on the GDS scores, two participants were suggestive of depression (GDS score $>5$ ) [17]. As for physical activity, women reported exercising about one hour a day on average, while men reported an hour and a half each day.
Results of partial correlations between static and dynamic balance variables for women and for men are presented in Tables 4 and 5, respectively. As can be seen, there are very few parameters that show significant correlations. For women, correlations between dynamic balance and static balance were found mainly with static parameters that are correlated with the vestibular system (frequency ranges of sway intensities F2, F3, and F4, and conditions HR, HL, $\mathrm{HB}$, and $\mathrm{HF}$ ). For men, the only significant correlations were found between the static balance parameters in eyes-opened conditions and the FR scores, while no significant correlations were found between the static balance parameters and the TUG scores.

Results of the regressions of the static balance parameters for the TUG by gender are presented in Table 2. Based on this analysis, it was found that, for women, age significantly explained $22.1 \%$ of the variance of the TUG scores, and adding static balance parameters to the regression revealed explanations between $28.1 \%$ and $40.4 \%$ of the variance of the TUG scores. The frequency ranges of sway intensities that stood out were $F 2, F 3$, and $F 4$, which may be related to peripheral vestibular function. For men, age did not significantly explain the variance of the TUG scores, while static balance parameters explained between $9.5 \%$ and $31.2 \%$ of the variance of the TUG scores; however only two of the regressions were found to be significant.

Results of the regressions of the static balance parameters for FR by gender are presented in Table 3. Based on this analysis, it was found that for women age did not explain significantly the variance of the FR scores, while static balance parameters significantly explained between $14.6 \%$ and $24.1 \%$ of the variance of the FR scores, in five postural conditions: NC, PC, HL, HB, and HF. These conditions may be related to peripheral vestibular function, because no vision (eyes were closed) is presented in these conditions. For men, age significantly explained $12.8 \%$ of the variance in the FR scores, while age and static balance parameters significantly explained between $23.9 \%$ and $41.7 \%$ of the variance of the FR scores. 
TABLE 3: Regression of static balance parameters on FR, by gender.

\begin{tabular}{lcccc}
\hline \multirow{2}{*}{ Static balance condition } & \multicolumn{2}{c}{ Women } & \multicolumn{2}{c}{ Men } \\
& $R^{2}$ total & Variable included & $R^{2}$ total & Variable included \\
\hline NO & 6.8 & & 37.2 & Age $^{*}$ \\
NC & 22.0 & $F 4^{*}, F 8^{* *}$ & 41.7 & Age $^{*}, F 4^{*}$ \\
PO & 6.9 & & 27.4 & Age $^{*}$ \\
PC & 24.1 & $F 1^{*}, F 2^{*}, F 7^{* *}$ & 23.9 & Age $^{*}$ \\
HR & 13.7 & $F 4^{* *}, F 5^{*}$ & 19.3 & Age $^{*}$ \\
HL & 16.1 & $F 4^{* *}$ & 35.2 & Age $^{*}, F 4^{*}$ \\
HB & 14.6 & $F 6^{*}$ & 35.0 & Age $^{*}$ \\
HF & 22.8 & 21.3 & Age $^{*}$ \\
\hline
\end{tabular}

${ }^{*} p<0.05 ;{ }^{* *} p<0.01$. NO: normal open position: standing straight, with eyes open. NC: normal closed position: standing straight, with eyes closed. PO: pillows open: standing on pillows, with eyes open. PC: pillows closed: standing on pillows, with eyes closed. HR: head right: standing with the head turned right, with eyes closed. HL: head left: standing with the head turned left, with eyes closed. HB: head back: standing with head tilted backward at a 30-degree angle, with eyes closed. HF: head forward: standing with head tilted forward about 30 degrees, with eyes closed. F1-F8 represent the pattern of sway intensities at different frequency ranges, as shown by Fourier spectral analysis of the postural sway waves. $F 1=$ low, $F 2-F 4=$ low-medium, $F 5$ - $F 6=$ medium-high, and $F 7-F 8=$ high frequencies of the Fourier band.

\section{Discussion}

The purpose of the current study was to examine to what extent the ability to control balance in static situations can explain performance in dynamic balance situations. The main finding of the study implies that in general there are low associations between static balance, as measured by a posturography system, and dynamic balance, as measured by two dynamic tests (TUG and FR). This finding is similar to the results of Hrysomallis et al. [23], who found weak associations between static balance and dynamic balance among elite football players, the results of Shimada et al. [13] and Muehlbauer et al. [14], who found low correlations or no correlations between static and dynamic balance abilities among elderly, and the results of Pau et al. [24], who found that static balance and dynamic balance were scarcely correlated among people with multiple sclerosis.

In addition, the low correlations found in the current study are indirectly similar to results of Paillard et al. [25], who examined the influence of a walking program on static and dynamic balance (among other variables) for older adults. They found that the intervention significantly increased dynamic balance but not static balance; it was suggested that different conditions and interventions may influence dynamic balance but not static balance and vice versa.

The low correlation between the two types of balance can be explained to some extent by the different demands that are placed on the control systems. During static postures the body's center of mass moves slowly with small sways, due to small external forces acting upon the body, while during dynamic tasks, greater external forces are present and more changes in the environment occur, thus placing higher demands on all balance control systems $[23,26]$.

The second finding of the current study was that, for women, static balance parameters that are correlated with peripheral vestibular function explained, to some extent, the variance in performance on the TUG assessment. This finding can be justified by the fact that the TUG test is based on changing heights and directions of the body, changes that irritate both the semicircular canals and the otoliths of the vestibular organ. These irritations may appear during head position changes that occur during several conditions of the Tetrax stability test (i.e., the HR, HL, HB, and HF). It is possible that people with low sensitivity to head position changes, as a consequence of vestibular deterioration, may exhibit poor performance of tasks that include head and body position changes. As falls are known to occur most frequently during walking or during transitions from sitting/standing to walking when head acceleration is higher, reduced capabilities of the vestibular system may be the responsible system for falls [27]. Indeed, it was found that vestibular fault (such as symptoms of true vertigo and sensation of movement) is associated with higher risk of falling during simple tasks, such as rising up from a chair or changing body position [9].

It is worth mentioning that our study is based on a group of highly educated volunteers, relatively healthy and active, who may not represent the general population of older adults. On the other hand, the fact that they are healthy and active enables the assessment of balance without the potential moderating effects of diseases and/or medications. Furthermore, our results corroborate previous studies reporting low associations between static and dynamic balance in elderly populations $[13,14]$. It is therefore likely to assume that the conclusions of the current study reflect healthy older adults in general.

As many clinicians want to assess the risk of fall among the elderly, a combination of instrumental tests that includes both static and dynamic tasks, as well as questionnaires, should be used, in order to increase the accuracy of such predictions $[1,7,23]$. In addition, as it is widely recognized that older people suffer from a number of impairments [4], physical trainers or physical therapists should include different aspects of balance control, including both static and dynamic exercises, and treat individuals in accordance 
TABLE 4: Partial correlation between static and dynamic parameters for women, adjusted for age.

\begin{tabular}{|c|c|c|}
\hline \multirow{2}{*}{ Static variables } & \multicolumn{2}{|c|}{ Dynamic variables } \\
\hline & TUG & FR \\
\hline NO F1 & .092 & .022 \\
\hline NO F2 & .106 & .076 \\
\hline NO F3 & -.113 & .040 \\
\hline NO F4 & .163 & .017 \\
\hline NO F5 & .125 & -.025 \\
\hline NO F6 & .172 & -.132 \\
\hline NO F7 & .070 & -.064 \\
\hline NO F8 & -.114 & .049 \\
\hline NO ST & .160 & -.082 \\
\hline NC F1 & -.078 & .169 \\
\hline NC F2 & -.057 & .000 \\
\hline NC F3 & .209 & .056 \\
\hline NC F4 & $.289^{*}$ & -.215 \\
\hline NC F5 & .098 & -.021 \\
\hline NC F6 & .073 & -.016 \\
\hline NC F7 & .014 & -.056 \\
\hline NC F8 & -.143 & .212 \\
\hline NC ST & .092 & -.110 \\
\hline PO F1 & -.051 & .061 \\
\hline PO F2 & .159 & .006 \\
\hline PO F3 & $.278^{*}$ & -.017 \\
\hline PO F4 & .187 & -.028 \\
\hline PO F5 & .038 & -.139 \\
\hline PO F6 & .103 & -.033 \\
\hline PO F7 & -.075 & -.148 \\
\hline PO F8 & -.125 & .018 \\
\hline PO ST & .105 & -.157 \\
\hline PC F1 & -.045 & -.183 \\
\hline PC F2 & .009 & -.004 \\
\hline PC F3 & .097 & -.179 \\
\hline PC F4 & -.030 & -.101 \\
\hline PC F5 & .105 & -.097 \\
\hline PC F6 & .148 & -.084 \\
\hline PC F7 & .041 & $-.310^{*}$ \\
\hline PC F8 & $.309^{*}$ & -.131 \\
\hline PC ST & .069 & -.185 \\
\hline HR F1 & .005 & .003 \\
\hline HR F2 & $.227^{*}$ & -.108 \\
\hline HR F3 & $.234^{*}$ & -.090 \\
\hline HR F4 & .215 & $-.273^{*}$ \\
\hline HR F5 & -.029 & -.191 \\
\hline HR F6 & -.073 & -.157 \\
\hline HR F7 & .039 & $-.282^{*}$ \\
\hline HR F8 & .042 & -.118 \\
\hline HR ST & .043 & -.221 \\
\hline HL F1 & .155 & -.047 \\
\hline
\end{tabular}

TABLE 4: Continued.

\begin{tabular}{|c|c|c|}
\hline \multirow{2}{*}{ Static variables } & \multicolumn{2}{|c|}{ Dynamic variables } \\
\hline & TUG & FR \\
\hline HL F2 & .145 & .013 \\
\hline HL F3 & .083 & -.077 \\
\hline HL F4 & $.234^{*}$ & -.216 \\
\hline HL F5 & .159 & -.014 \\
\hline HL F6 & .039 & -.102 \\
\hline HL F7 & .030 & -.186 \\
\hline HL F8 & .011 & .156 \\
\hline HL ST & .112 & -.206 \\
\hline HB $F 1$ & .116 & .014 \\
\hline HB F2 & $.236^{*}$ & -.017 \\
\hline HB F3 & .015 & -.028 \\
\hline HB F4 & .213 & -.223 \\
\hline HB F5 & .172 & -.083 \\
\hline HB F6 & .096 & .032 \\
\hline HB F7 & .090 & -.113 \\
\hline HB F8 & $.259^{*}$ & -.086 \\
\hline HB ST & .119 & -.126 \\
\hline HF $F 1$ & .073 & .113 \\
\hline HF F2 & .162 & -.043 \\
\hline HF F3 & .102 & -.162 \\
\hline HF F4 & .139 & $-.282^{*}$ \\
\hline HF F5 & .075 & $-.333^{*}$ \\
\hline HF F6 & -.002 & -.059 \\
\hline HF F7 & .072 & $-.344^{*}$ \\
\hline HF F8 & -.180 & -.012 \\
\hline HF ST & .115 & $-.268^{*}$ \\
\hline
\end{tabular}

with their specific impairments [8]. In addition, based on the second finding of the current study, trainers should pay great attention to the performance of their patients during activities that involve direction or height changes.

\section{Conclusions}

Based on the poor associations between static and dynamic balance found in the current study, it should be noted that essential information might be overlooked if only one component is measured. This may be particularly relevant to prospective longitudinal studies investigating the relationship between balance ability and the risk of falling among the elderly.

In addition, for preservation and rehabilitation purposes, physical activity trainers should consider the inclusion of both static and dynamic balance exercises in training programs for older adults. 
TABLE 5: Partial correlation between static and dynamic parameters for men, adjusted for age.

\begin{tabular}{|c|c|c|}
\hline \multirow{2}{*}{ Static variables } & \multicolumn{2}{|c|}{ Dynamic variables } \\
\hline & TUG & FR \\
\hline NO F1 & .010 & .295 \\
\hline NO F2 & .107 & .027 \\
\hline NO F3 & .043 & -.048 \\
\hline NO F4 & -.069 & $.366^{*}$ \\
\hline NO F5 & .063 & .328 \\
\hline NO F6 & .022 & $.407^{*}$ \\
\hline NO $F 7$ & .010 & .229 \\
\hline \multicolumn{3}{|l|}{ NO F8 } \\
\hline NO ST & .070 & $.344^{*}$ \\
\hline $\mathrm{NC} F 1$ & -.173 & .336 \\
\hline $\mathrm{NC} F 2$ & -.214 & .141 \\
\hline NC F3 & .146 & .207 \\
\hline NC F4 & .238 & -.122 \\
\hline NC F5 & -.006 & .191 \\
\hline NC F6 & -.076 & .018 \\
\hline NC F7 & -.044 & .186 \\
\hline NC F8 & -.072 & .131 \\
\hline NC ST & .046 & .104 \\
\hline PO $F 1$ & .165 & -.035 \\
\hline PO F2 & .052 & -.024 \\
\hline PO F3 & .030 & -.167 \\
\hline PO F4 & .083 & -.203 \\
\hline PO F5 & .077 & -.271 \\
\hline PO F6 & .181 & -.224 \\
\hline PO F7 & .292 & -.338 \\
\hline \multicolumn{3}{|l|}{ PO F8 } \\
\hline PO ST & .191 & $-.419^{*}$ \\
\hline PC F1 & -.030 & .241 \\
\hline PC F2 & .053 & .191 \\
\hline PC F3 & -.265 & .072 \\
\hline PC F4 & .003 & -.052 \\
\hline PC F5 & -.071 & .065 \\
\hline PC F6 & .098 & .070 \\
\hline PC F7 & .148 & .056 \\
\hline PC F8 & -.011 & .096 \\
\hline PC ST & .079 & .018 \\
\hline HR $F 1$ & .174 & -.016 \\
\hline HR F2 & -.072 & -.038 \\
\hline HR F3 & .016 & .120 \\
\hline HR F4 & .012 & -.016 \\
\hline HR F5 & -.124 & -.011 \\
\hline HR F6 & -.062 & -.088 \\
\hline HR F7 & .223 & -.148 \\
\hline HR F8 & -.045 & -.043 \\
\hline HR ST & .058 & -.135 \\
\hline HL $F 1$ & .036 & .213 \\
\hline
\end{tabular}

TABLE 5: Continued.

\begin{tabular}{|c|c|c|}
\hline \multirow{2}{*}{ Static variables } & \multicolumn{2}{|c|}{ Dynamic variables } \\
\hline & TUG & FR \\
\hline HL F2 & -.090 & .140 \\
\hline HL F3 & -.139 & .102 \\
\hline HL F4 & -.173 & -.041 \\
\hline HL F5 & -.004 & -.063 \\
\hline HL F6 & -.054 & -.084 \\
\hline HL F7 & .027 & .026 \\
\hline \multicolumn{3}{|l|}{ HL F8 } \\
\hline HL ST & -.047 & -.039 \\
\hline HB $F 1$ & -.123 & .325 \\
\hline HB F2 & -.004 & .321 \\
\hline HB F3 & .054 & .086 \\
\hline HB F4 & .094 & .185 \\
\hline HB F5 & .138 & .221 \\
\hline HB F6 & .014 & .166 \\
\hline HB F7 & .086 & .206 \\
\hline HB F8 & -.065 & .203 \\
\hline HB ST & .069 & .202 \\
\hline HF F1 & -.096 & .158 \\
\hline HF F2 & .026 & .183 \\
\hline HF F3 & .024 & .113 \\
\hline HF F4 & -.159 & .200 \\
\hline HF F5 & .035 & .254 \\
\hline HF F6 & -.048 & .194 \\
\hline HF F7 & .074 & .181 \\
\hline HF F8 & -.029 & -.086 \\
\hline HF ST & .009 & .218 \\
\hline
\end{tabular}

\section{Conflicts of Interest}

The authors declare that there are no conflicts of interest regarding the publication of this paper.

\section{Acknowledgments}

The authors would like to acknowledge the valuable contributions of Dina Olswang for reviewing and editing the final version of the manuscript.

\section{References}

[1] M. T. Karimi and S. Solomonidis, "The relationship between parameters of static and dynamic stability tests," Journal of Research in Medical Sciences, vol. 16, no. 4, pp. 530-535, 2011.

[2] C. Rival, H. Ceyte, and I. Olivier, "Developmental changes of static standing balance in children," Neuroscience Letters, vol. 376, no. 2, pp. 133-136, 2005.

[3] E. G. da Silva Borges, R. G. de Souza Vale, S. A. Cader et al., "Postural balance and falls in elderly nursing home residents 
enrolled in a ballroom dancing program," Archives of Gerontology and Geriatrics, vol. 59, no. 2, pp. 312-316, 2014.

[4] S. R. Lord, J. A. Ward, P. Williams, and K. J. Anstey, "Physiological factors associated with falls in older community-dwelling women," Journal of the American Geriatrics Society, vol. 42, no. 10, pp. 1110-1117, 1994.

[5] S.-I. Cho and D.-H. An, "Effects of a fall prevention exercise program on muscle strength and balance of the Old-old elderly," Journal of Physical Therapy Science, vol. 26, no. 11, pp. 1771-1774, 2014.

[6] A. Desai, V. Goodman, N. Kapadia, B. L. Shay, and T. Szturm, "Relationship between dynamic balance measures and functional performance in community-dwelling elderly people," Physical Therapy, vol. 90, no. 5, pp. 748-760, 2010.

[7] A. Soto-Varela, A. Faraldo-García, M. Rossi-Izquierdo et al., "Can we predict the risk of falls in elderly patients with instability?” Auris Nasus Larynx, vol. 42, no. 1, pp. 8-14, 2015.

[8] F. B. Horak, "Postural orientation and equilibrium: What do we need to know about neural control of balance to prevent falls?" Age and Ageing, vol. 35, no. 2, pp. ii7-iill, 2006.

[9] E. Tuunainen, J. Rasku, P. Jäntti, and I. Pyykkö, "Risk factors of falls in community dwelling active elderly," Auris Nasus Larynx, vol. 41, no. 1, pp. 10-16, 2014.

[10] H. Razavi, "A Comparison between Static and Dynamic Stability in Postural Sway and Fall Risks," Journal of Ergonomics, vol. 07, no. 01, 2016.

[11] N. D. Carter, K. M. Khan, A. Mallinson et al., "Knee extension strength is a significant determinant of static and dynamic balance as well as quality of life in older community-dwelling women with osteoporosis," Gerontology, vol. 48, no. 6, pp. 360368, 2002.

[12] D. J. Mayson, D. K. Kiely, S. I. LaRose, and J. F. Bean, "Leg strength or velocity of movement: Which is more influential on the balance of mobility limited elders?" American Journal of Physical Medicine and Rehabilitation, vol. 87, no. 12, pp. 969976, 2008.

[13] H. Shimada, S. Obuchi, N. Kamide, Y. Shiba, M. Okamoto, and S. Kakurai, "Relationship with dynamic balance function during standing and walking," American Journal of Physical Medicine and Rehabilitation, vol. 82, no. 7, pp. 511-516, 2003.

[14] T. Muehlbauer, C. Besemer, A. Wehrle, A. Gollhofer, and U. Granacher, "Relationship between strength, power and balance performance in seniors," Gerontology, vol. 58, no. 6, pp. 504-512, 2012.

[15] M. F. Folstein, S. E. Folstein, and P. R. McHugh, “"Mini mental state". A practical method for grading the cognitive state of patients for the clinician," Journal of Psychiatric Research, vol. 12, no. 3, pp. 189-198, 1975.

[16] M. P. Lawton and E. M. Brody, "Assessment of older people: self-maintaining and instrumental activities of daily living," The Gerontologist, vol. 9, no. 3, pp. 179-186, 1969.

[17] T. L. Brink, J. A. Yesavage, O. Lum, P. H. Heersema, M. Adey, and T. L. Rose, "Screening tests for geriatric depression," Clinical Gerontologist, vol. 1, no. 1, pp. 37-43, 1982.

[18] D. Podsiadlo and S. Richardson, "The timed "Up and Go": a test of basic functional mobility for frail elderly persons," Journal of the American Geriatrics Society, vol. 39, no. 2, pp. 142-148, 1991.

[19] P. W. Duncan, D. K. Weiner, J. Chandler, and S. Studenski, "Functional reach: a new clinical measure of balance," The Journals of Gerontology, vol. 45, no. 6, pp. 192-197, 1990.
[20] R. Kohen-Raz, "Application of tetra-ataxiametric posturography in clinical and developmental diagnosis.," Perceptual and Motor Skills, vol. 73, no. 2, pp. 635-656, 1991.

[21] N. Akkaya, N. Doganlar, E. Çelik et al., "Test-retest reliability of Tetrax ${ }^{\circledR}$ static posturography system in young adults with low physical activity level," vol. 10, no. 6, pp. 893-900, 2015.

[22] D. Ryan and M. Heaner, "Guidelines (2013) for managing overweight and obesity in adults. Preface to the full report," Obesity (Silver Spring, Md.), vol. 22, pp. S1-S3, 2014.

[23] C. Hrysomallis, P. McLaughlin, and C. Goodman, "Relationship between static and dynamic balance tests among elite Australian Footballers," Journal of Science and Medicine in Sport, vol. 9, no. 4, pp. 288-291, 2006.

[24] M. Pau, M. Porta, G. Coghe et al., "Are static and functional balance abilities related in individuals with Multiple Sclerosis?" Multiple Sclerosis and Related Disorders, vol. 15, pp. 1-6, 2017.

[25] T. Paillard, C. Lafont, M. C. Costes-Salon, D. Rivière, and P. Dupui, "Effects of brisk walking on static and dynamic balance, locomotion, body composition, and aerobic capacity in ageing healthy active men," International Journal of Sports Medicine, vol. 25, no. 7, pp. 539-546, 2004.

[26] D. A. Winter, F. Prince, J. S. Frank, C. Powell, and K. F. Zabjek, "Unified theory regarding A/P and M/L balance in quiet stance," Journal of Neurophysiology, vol. 75, no. 6, pp. 2334-2343, 1996.

[27] E. Anson and J. Jeka, "Perspectives on aging vestibular function," Frontiers in Neurology, vol. 6, article no. 269, 2016. 


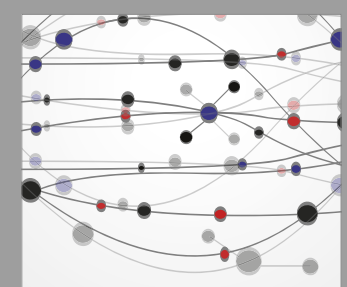

The Scientific World Journal
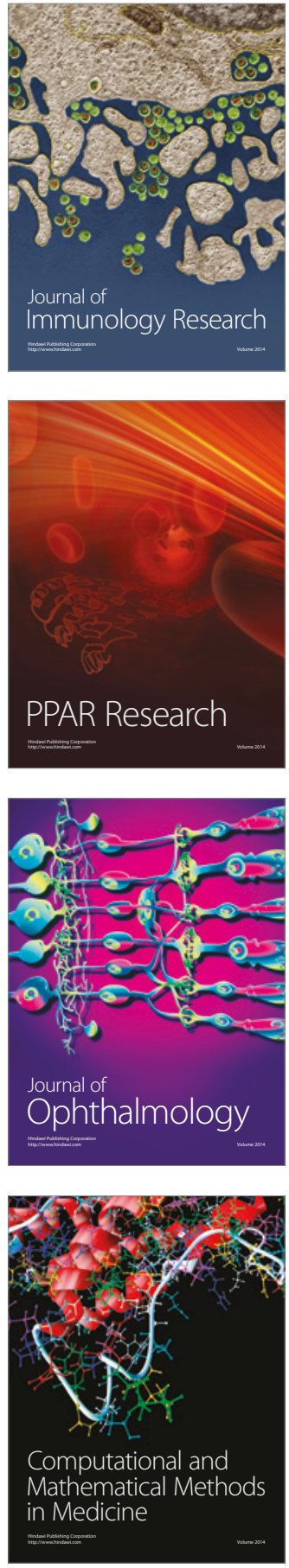

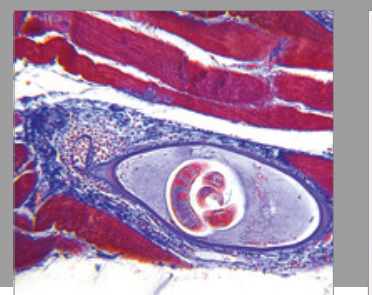

Gastroenterology Research and Practice
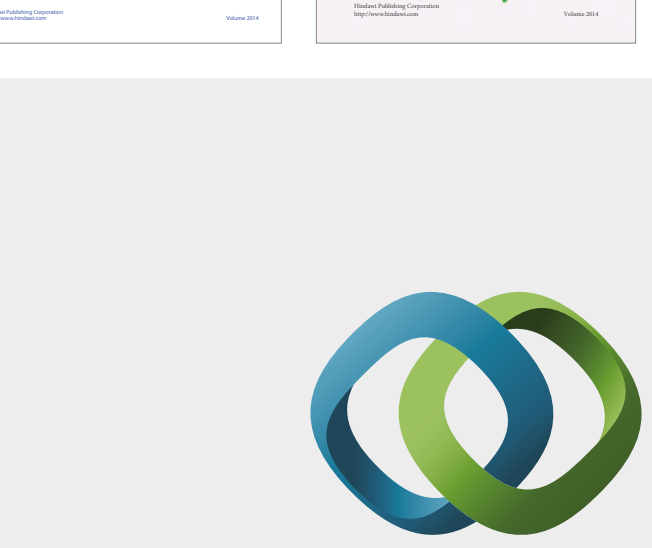

\section{Hindawi}

Submit your manuscripts at

https://www.hindawi.com
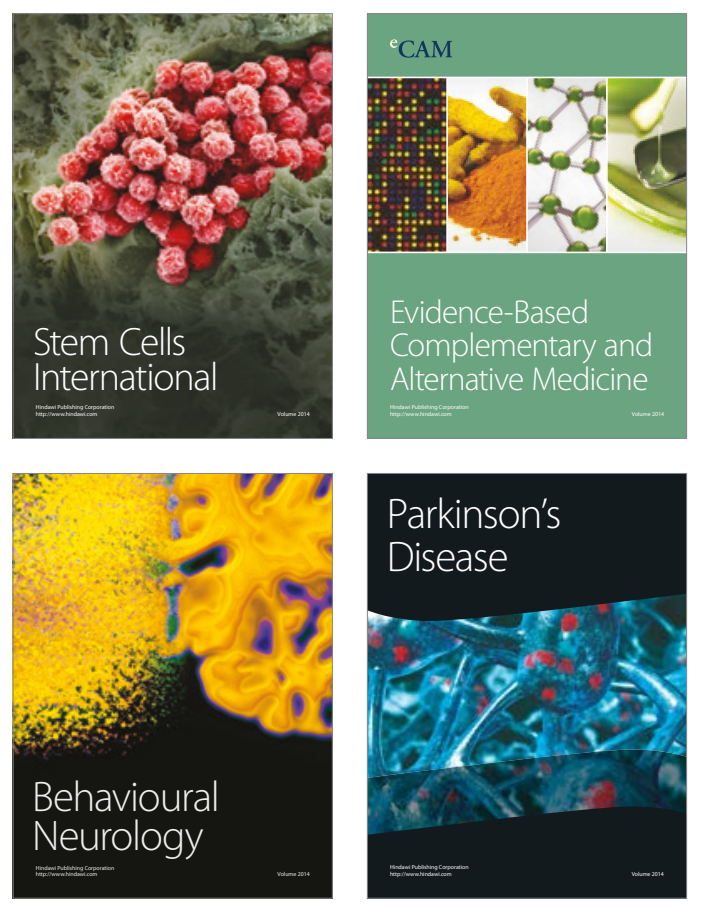
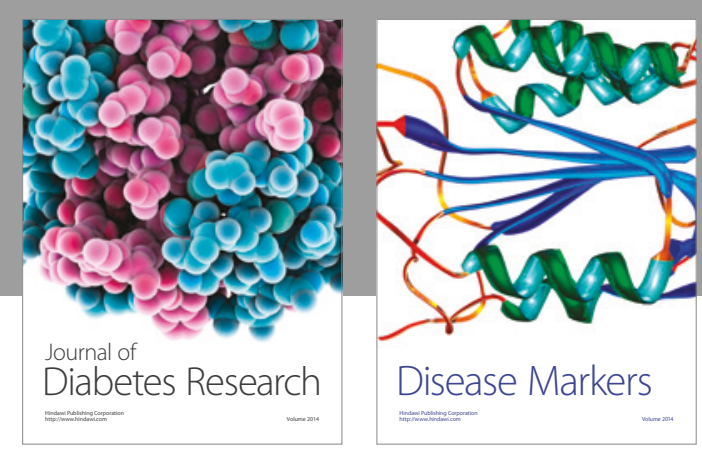

Disease Markers
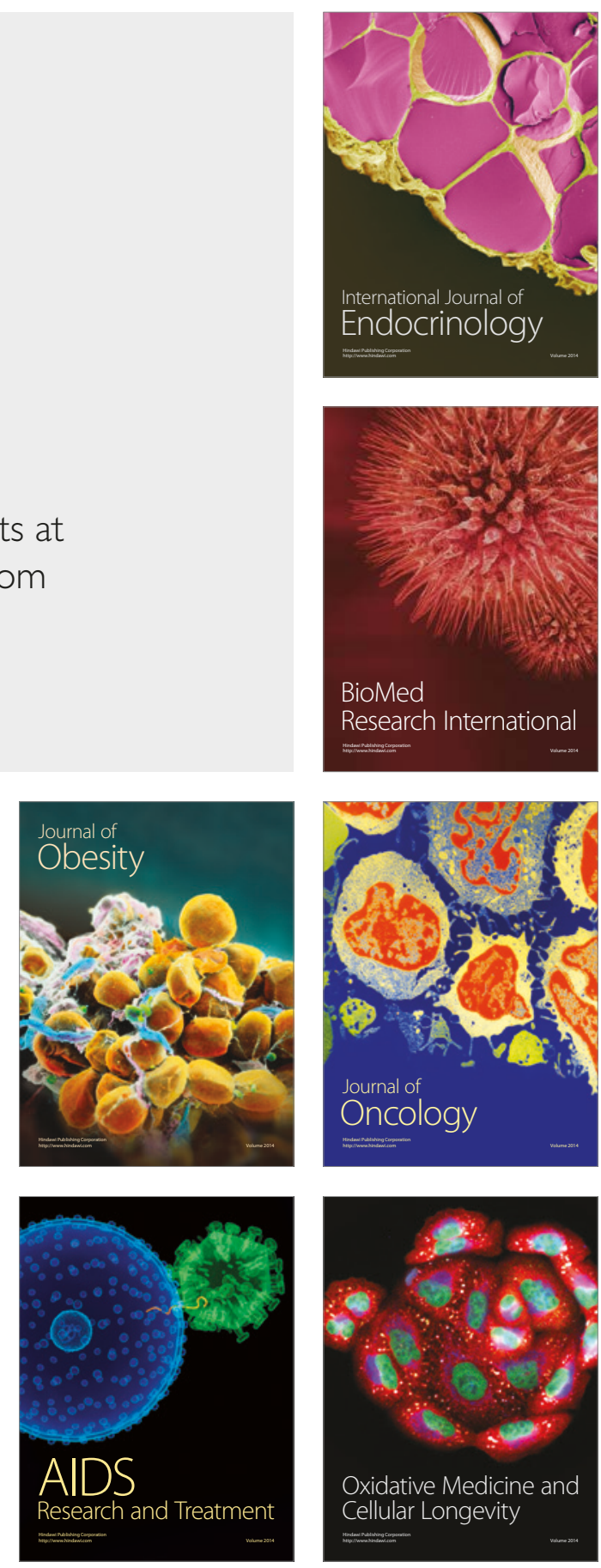